\title{
Configuration, Deployment, and Scheduling Models for Management and Optimization of Patrol Services
}

\author{
Bin Yang, Zhi-Hua Hu, and Jing-Xian Zhou \\ Logistics Research Center, Shanghai Maritime University, Shanghai 200135, China \\ Correspondence should be addressed to Zhi-Hua Hu; zhhu@shmtu.edu.cn
}

Received 30 September 2014; Revised 15 November 2014; Accepted 27 November 2014

Academic Editor: Kishin Sadarangani

Copyright (C) 2015 Bin Yang et al. This is an open access article distributed under the Creative Commons Attribution License, which permits unrestricted use, distribution, and reproduction in any medium, provided the original work is properly cited.

\begin{abstract}
This paper presents a decision support system (DSS) and its models for patrol service center (PSC). PSC plays an important role in public security and emergency management. The configuration, deployment, and scheduling of resources of PSC are important for improving the efficiency of patrol-related resources, service quantity, and emergency response capability. A series of decisionmaking models of the DSS are studied. First, the criteria and models are proposed for configuring and deploying PSCs; second, three types of models for incremental, direct, and redeployment optimization are built in views for decisions aiming at PSC configuration, deployment, and scheduling problems; third, considering three typical patrol-related service scenarios (alarm assignment, main road blockade, and besiege program), three scheduling models are built, respectively, for PSC-related service and coordination of multiple PSCs. This work contributes to the literature on patrol services and network optimization problems in the following aspects: based on a series of models, a DSS framework is designed for PSCs; the models are formulated for resource management and scheduling upon geography information system; coordination strategies among close PSCs are incorporated into decision models. These features are examined in integration manners. The assessment criteria and optimization models studied in the paper are beneficial for building DSSs for PSC.
\end{abstract}

\section{Introduction}

Police patrolling is regarded as one of the best well-known practices for implementing public-safety preventive policies towards the combat of an assortment of urban crimes [1]. In China, patrol police provides emergency service for local public security and contingent events. A patrol department is a police unit created primarily for the purpose of overseeing and enforcing traffic safety compliance on roads and highways. There are many managerial problems for the configuration, deployment, and scheduling of patrol-related resources responding to contingent events. To implement these functions more effectively, some patrol service centers (PSCs) are gradually established in major traffic arteries and densely inhabited districts. Due to the shortage of police resources, how can the PSCs be deployed, how can the area of each PSC be assigned, and how can the patrol-related resource be scheduled are practical problems faced by patrol management departments.
Activated by the requirements on PSC information management systems in China and the problems studied in the literature, a decision support system (DSS) for PSC with the following decision-making problems is identified: the configuration, deployment, reconfiguration, and redeployment of PSCs; coordinative response for serious contingent events; emergency blockade for serious suspects; and regional besiege program for serious suspects. The above problems, their optimization models, and decision strategies should be studied based on spatial information. In this study, a general DSS framework is proposed for PSC.

In the literature, some models have been proposed for patrol-elated optimization problems. Coupe and Blake [2], D'Amico et al. [3], Lau et al. [4], and Lou et al. [5] deal with the configuration and deployment problems of patrol-related resources in strategic levels. Keskin et al. [6] and Yin [7, 8] studied the scheduling problems of patrol-related resources and tasks. Various models were studied independently such that the practical values of them are limited. The following 
literature studied the optimization problems of patrol-related resources in strategic levels. Pal and Sinha [9] evaluated freeway service patrol system configurations by using simulation. Bodily [10] proposed a decision-making model for a resource allocation problem in the design of service areas for police mobile units. Using multiattribute utility theory, alternative designs were evaluated according to the preferences for efficiency and equality of service of three interest groups: citizens, police, and administrators. Meaningful measures of inequality were developed and an algorithm was created for generating improved sector designs. Carroll and Laurin [11] defined police patrol zones by computer simulation. The criterion is to maximize the amount of time available for patrol duties; the constraints are the current average time to respond to a call and a limit on the number of patrol cars. Kern [12] allowed customized sector design and deployment strategies to be tested by simulation. The simulation model reported allows the administrator to study the performance of several complex dispatching tactics including multiple unit dispatching, preemption, and intersector dispatching. D'Amico et al. [3] proposed a simulated annealing algorithm for districting the police commands. Coupe and Blake [2] studied the effects of patrol workloads of and response strength to burglary emergencies. Curtin et al. [13] presented a new method for determining efficient spatial distributions of police patrol areas. This method employs a traditional maximal covering formulation and an innovative backup covering formulation to provide alternative optimal solutions to police decision makers. Assunção and Furtado [14] describe a heuristic method based on graph partitioning algorithms with the purpose of improving the demarcation of areas for police patrolling. Lou et al. [5] investigated the problem of deploying freeway service patrols to detect, respond to, and clear traffic incidents in deterministic and stochastic settings. Lau et al. [4] developed a mathematical model to improve the deployment of security guards in a company located in south China for the purpose of immediate response to complaints.

The second type of topics is about scheduling patrolrelated resources and tasks. Khattak et al. [15] developed an approach to help determine the most beneficial locations for patrol deployment by using expanded placement criteria. Results of the research were incorporated into a decision support tool that allows easy planning and operational assessment of candidate sites by comparing performance values between sites, modeling the effect of patrol services, and estimating their key potential benefits. Yin [7] proposed a min-max bilevel programming model to find an optimal fleet allocation solution for freeway service patrols. The model minimizes the maximal traveling time when incidents may incur, which was solved by a heuristic algorithm. Reis et al. [1] devised an evolutionary multiagent-based simulation tool to assist police managers in the design of effective police patrol route strategies, where the high crime-density regions are well covered by routine patrol surveillance. Keskin et al. [6] addressed the problem of determining the routes of state troopers to maximize the coverage of highway spots with high frequencies of crashes. A specific mixed-integer linear programming (MIP) model was developed for this problem under time restrictions and budget limitations. An algorithm incorporating heuristics based on local search and tabu search is designed to solve the model. Yin [8] formulated a mixed-integer nonlinear programming model based on scenario analysis for the fleet allocation problem to achieve faster response and reduced clearance time in patrol-related management background. Chaiken and Dormont [16, 17] developed advanced patrol car allocation models, which were updated to include multiple dispatch queuing [18], and the validity was tested with generally positive results given the limitations of a model dependent on human behavior [19]. Further, Sacks [20] examined the spatial deployment of police cars with respect to four criteria: response time, workload balance, patrol frequency, and interdistrict dispatches.

Comparing to the above literature, the contributions of this study include the following points. First, a DSS framework is designed for PSCs. Second, mathematical models are proposed for resource management and scheduling upon geography information system (GIS). Third, coordination strategies among close PSCs are incorporated into decision models.

The paper is organized as follows. In Section 2, a framework of DSS for PSC is proposed. In Section 3, the assessment criteria and a series of decision models in the DSS PSCs are studied. In Section 4, the models are demonstrated by using the sample data of a city in China. In Section 5, the conclusion and the suggestions of future work are discussed.

\section{System Framework}

The configuration, deployment, and scheduling of PSCs depend on three types of information: (1) positions of roads and residents; (2) real-time positions and status of patrolrelated resources, for example, cars and phones; (3) the urban public security system and other management systems and the collected and transferred information by the 110 call center. The accuracy and intervals of the three types of realtime information impose a great effect on the efficiency and performance of scheduling the PSCs. Moreover, the historical information of these types is the premise for deploying PSCs. As shown in Figure 1, the DSS for PSC (PSC DSS) is built upon spatial data from GIS, the databases of the city and PSCs, and other information management systems. PSC DSS connects with the public security inspection and management system and 110 call center, to acquire the service demands which are sent to specific PSC by wireless communication.

The PSC DSS in Figure 1 includes two parts of decision models: scheduling models which usually focus on real-time operations and deployment models which are commonly periodically performed and have strong strategic significance. For scheduling models, the service area assignment is also performed periodically. According to the alarm demands assigned to specific PSC, specific scheduling model is chosen to be performed. Emergent blockade and besiege programs represent two scheduling levels. Emergent blockade aims for the main crossroads in a specific areas in the city when some serious suspects are escaping; the besiege program is to schedule the patrol-related resources in the urban area for the escaping serious suspects. These two scheduling 


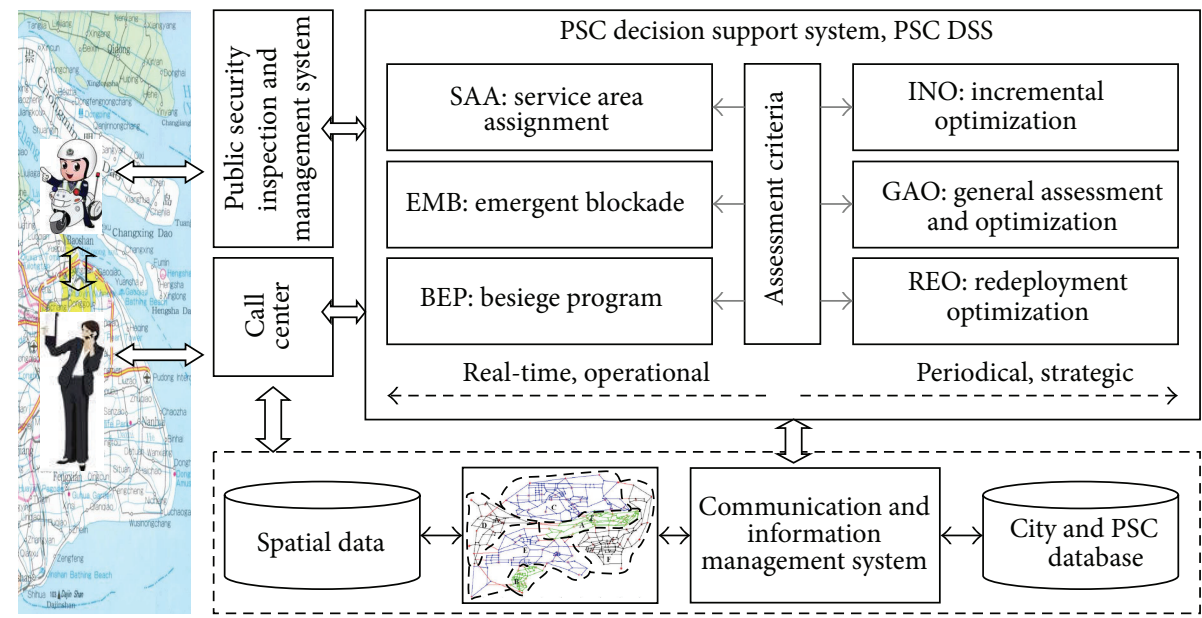

FIGURE 1: The proposed framework of PSC DSS.

models coordinate the PSCs. Three models are designed for deploying PSCs. First, according to the patrol service rules and general requirements of patrol-related resource configuration, a system of assessment criteria is proposed. Second, based on the current deployment of PSCs, new PSCs are incrementally built to enhance the service capability in the areas with more alarms. The third is about redeploying PSCs. It is apparent that both the incremental deployment and redeployment aim at finding optimal solutions under the assessment criteria.

\section{Models}

In the following, the problems proposed above and their decision models proposed in Figure 1 are studied.

3.1. Service Area Assignment. The model of service area assignment (SAA) is to allocate crossroads to PSCs after the alarm demands are all ascribed to crossroads by GIS. SAA has two typical scenarios. First, the crossroads with workloads represented by alarms are periodically assigned to PSCs. Second, the service demand triggered by an alarm is sent to a specific PSC by 110 call center according to the location of the alarm. The processes in the above scenarios should utilize the information that can be returned by the following systems: urban information management system, urban GIS, and 110 call center.

SAA assigns nodes in the traffic network to PSC. The fundamental data that can be utilized include the crossroad node set that can be obtained by topology analysis based on GIS and the cost matrix among these nodes; the set of PSCs and the nodes of all PSCs; the alarm frequency and the workload of each node that can be calculated from the patrol service records; finally the length of time promised for responding to alarm by patrol service after the alarm is accepted. The length is three minutes in many cities in China. According to the above information, the objective is to build a map from road nodes to PSCs, where a PSC can serve a set of nodes.
The related sets, parameters, and decision variables are defined as follows. The road node set is denoted by NOD. The PSC set is $S, S \subseteq$ NOD. $C T_{a, b}$ sets the traveling time between two nodes $a, b \in$ NOD. $F_{n}$ sets the alarm frequency of node $n \in$ NOD. The response time promised by PSC is denoted by $M T$, and commonly $M T=3$. Four decision variable groups are defined below. First, $x_{s, n}=1$, if the alarm from the $n \in \mathrm{NOD}$ is processed by the PSC located at $s \in \mathrm{NOD}$; or else zero. Second, $s t p_{s}$ defines the workload of the PSC $(s \in S)$. Third, $n t r_{n}$ denotes the response time of the alarm proposed at the node $n \in \mathrm{NOD}$. The fourth group of decision variables is defined for improving the readability of the model: stpma is the maximal workload of all PSCs; stpmi is the minimal workload of all PSCs; $n t r m a$ is the maximal weighted response time of all nodes; and ntrall is the total weighted response time of all nodes.

The objective functions reflect the optimization and balance of the benefits of PSC and residents. For a PSC, the total service workloads represented by the service times and service distances are minimized. Among different PSCs, the workloads should be balanced. For the residents, the response time to alarm is most important. The promise of three minutes for response should be tried to be satisfied. In the following, (1)-(3) are designed to cater for these objectives.

(1) Balance the total working time computed by weighted alarm frequencies.

This objective is to minimize the difference between the maximal workload and the minimal workload (1), where the maximal workload and the minimal workload are calculated by (8) and (9). From the views of optimal response and service quantity, two other objectives are defined in the following.

(2) Minimize the maximal weighted response time of nodes.

Equation (2) minimizes the longest weighted response time, where ntrma is defined further by (7).

(3) Minimize the total weighted response time. 
Equation (2) is defined in the view of minimizing the service workload of a single node. From another view, (3) minimizes the weighted response service workloads of all nodes, where $n$ trall is defined by (10).

Before defining the variables used in the objective functions, the response time for node's alarm and the total weighted service time for each PSC are defined. In (4), according to the assignment of nodes to PSCs, the response time for each node is defined. According to the promise of response time within three minutes, if it is a hard constraint, the response time of each node should satisfy (5), where MT is the limit.

Based on the constraints ((4)-(6)), the objectives ((1)(3)) are further defined by (7)-(10). The maximal response time of node is defined by (7). The maximal workload of PSC is computed by (8), whereas (9) calculates the minimal workloads for PSC. The total weighted response time of all nodes is further summed by (10).

Additionally, the constraint in (11) sets a restriction that the alarm from a node must and can only be assigned to a PSC. Moreover, for simplicity, the node of a PSC should be assigned to this PSC (12):

$$
\begin{gathered}
\min f_{1}=s t p m a-s t p m i, \\
\min f_{2}=n t r m a, \\
\min f_{3}=n t r a l l, \\
n t r_{n}=\sum_{s \in S}\left(x_{s, n} \cdot C T_{s, n}\right), \quad \forall n \in \mathrm{NOD}, \\
n t r_{n} \leq M T, \quad \forall n \in \mathrm{NOD}, \\
\text { stps }_{s} \sum_{n \in \mathrm{NOD}}\left(x_{s, n} \cdot C T_{s, n} \cdot F_{n}\right), \quad \forall s \in S, \\
n t r m a \geq n t r_{n}, \quad \forall n \in \mathrm{NOD}, \\
s t p m a \geq s t p_{s}, \quad \forall s \in S, \\
s t p m i \leq s t p_{s}, \quad \forall s \in S, \\
n t r a l l=\sum_{n \in \mathrm{NOD}}\left(n t r_{n} \cdot F_{n}\right), \\
\sum_{s \in S} x_{s, n}=1, \quad \forall n \in \mathrm{NOD}, \\
x_{s, s}=1, \quad \forall s \in S .
\end{gathered}
$$

3.2. Emergent Blockade. Emergent blockade (EMB) is usually a solution to deal with or prevent the accidents in the following cases: when there is big accident that happened in the area, and the car flow and people flow will impede solving the problem, for example, a traffic accident by dangerous chemicals or virus; the flows of cars and people themselves will incur accidents, for example, big fog climate which requires blockading highways. The model EMB builds a map between the main road nodes and the patrol-related resources.
The quantity of main road nodes is presumed to be less than the quantity of PSCs. EMB assigns main road nodes to PSCs. Therefore, three set types are involved: the road nodes, PSCs, and the main road nodes. The parameters mainly include a matrix of traveling time between road nodes. The decision variables include the mapping relations between the main road nodes and PSCs. Besides NOD and $S, E$ defines the node set attached to the PSCs, $E \subseteq$ NOD. The variable $x_{s, e}=1$, if the main road node $e \in E$ is blockaded by PSC $s \in S$; or else 0 . et $t_{e}$ is the time to blockade the main road node $e \in E$. etma represents the maximal time to blockade the main road nodes, whereas etall is the total time to blockade all main road nodes.

The objectives of blockade should emphasize urgency. The urgency here has two meaning levels: the blockade should be finished as soon as possible; the blockade cost should be minimized by optimizing the assignment of PSCs to main road nodes. etma has been defined to denote the maximal time of blockade, and etall is the total time of blockade. Then, two objectives are defined ((13) and (14)) to minimize etma and etall. They are further computed by (18) and (19).

The constraint functions in EMB include two parts: assigning PSCs to the main road nodes and the variables used in the objectives:

(1) every main road node should be assigned a PSC for blockade (15);

(2) every PSC cannot take charge of more than one main road node (16);

(3) the time to blockade a given main road node is computed by $x(17)$;

(4) the maximal time of blockade is limited ((18) and (19));

$$
\begin{gathered}
\min f_{1}=\text { etma, } \\
\min f_{2}=\text { etall, } \\
\sum_{s \in S} x_{s, e}=1, \quad \forall e \in E, \\
\sum_{e \in E} x_{s, e} \leq 1, \quad \forall s \in S, \\
\text { et } e_{e}\left(x_{s, e} \cdot C T_{s, e}\right), \quad \forall e \in E, \\
\text { etma } \geq e t_{e}, \quad \forall e \in E, \\
\text { etall }=\sum_{e \in E} \text { et } t_{e} .
\end{gathered}
$$

3.3. Besiege Program. Designing besiege program (BEP) is a dynamic optimization problem. Considering a serious case happens at node $P \in \mathrm{NOD}$, the suspect has escaped by car after the alarm was received after three minutes. When the escape direction of the suspect cannot be determined, if the PSC $s \in S$ decides to blockade the suspect at the node $a \in \mathrm{NOD},(20)$ should be satisfied to ensure that the patrol policemen can reach not before the suspect reaches it. 
In another aspect, the time of finishing besiege can determine the coverage of the besiege program. For example, if it is wished to finish besiege within five minutes, then every PSC should go to the nodes near to the node $P$. If the time is 10 minutes, the besiege scope should be larger. The smaller scope indicates that the patrol policemen may blockade the nodes that the suspect has passed. Therefore, the besiege program is transferred into the node selection problem in the set of nodes that can be reached within a period of time from the node $P$. In other words, it is to choose some nodes and determine the corresponding PSCs to blockade the suspect. Upon the road node set and the PSC set, $C N$ is introduced to represent the node set for blockade, whose elements are chosen by (21), where $T L$ denotes the time from when the suspect begins to escape at the node $P$ to the time when the besiege is finished. A primary parameter in BEP is CT. The decision variable $x_{s, n}$ denotes the assignment of the nodes of blockade to PSCs; $x_{s, n}=1$, when the PSC ( $s \in \mathrm{NOD}$ ) will blockade the node $(n \in C N)$; 0 , otherwise.

The assignment of PSC to blockaded node should satisfy the time within which the node is reachable (22). In the model proposed, each PSC can only blockade no more than one node (23). At the same time, each node can only be blockaded by no more than one PSC (24):

$$
\begin{gathered}
C T_{s, a}+3 \leq C T_{a, P}, \\
C N=\left\{n \in \mathrm{NOD} \mid C T_{n, P} \leq T L\right\}, \\
\left(C T_{s, n}+3\right) \cdot x_{s, n} \leq C T_{n, p}, \quad \forall s \in S, n \in C N, \\
\sum_{n \in C N} x_{s, n} \leq 1, \quad \forall s \in S, \\
\sum_{s \in S} x_{s, n} \leq 1, \quad \forall n \in C N .
\end{gathered}
$$

Based on the above constraints, in order to improve the efficiency of besiege, the patrol-related resources should be utilized fully. In other words, the quantity of the devoted PSCs should be maximized:

$$
\max f_{1}=\sum_{s \in S, n \in C N} x_{s, n}
$$

The above model does not consider embracement by the blockade node set to the node with contingent accidents. It can be supported by choosing nodes in $C N$ and building assessment criteria for the embracement, as indicated in Algorithm 1. Then, the above model can be applied to optimize the besiege program.

3.4. PSC Assessment Criteria. The quantity of PSCs and the positions of them have global impacts on the efficiency of the patrol-related resources and scheduling plans. The assessment of PSC involves the following aspects. First, from the view of assessed objectives, there are maximal, minimal, and other statistical measures of the quantity of PSCs and the total workloads. Second, the criteria may refer to PSC or to the nodes with alarms. Third, the criteria may measure the service efficiency from the view of PSC or the service satisfaction degree from the view of the residents.

In summary, about the configuration and deployment of PSCs, there are six criteria, which in fact have been indicated in the above studies:

(1) the degree of balance of the total working time weighted by the alarm frequency,

(2) the weighted response time for each node,

(3) the total weighted response time for all nodes,

(4) the total quantity of PSCs,

(5) the blockade time for each main road node,

(6) the total time of blockading all main road nodes.

3.5. Incremental Optimization. The incremental optimization (INO) of the quantities of PSCs is a practical optimization problem. Incremental optimization refers to the fact that the updating program may introduce new PSCs and determine their positions on the basis of the present situation. Obviously, the new set of PSCs should improve performance under the assessment criteria.

Besides the definitions above, the sets, parameters, and decision variables used in INO are defined as follows. The parameter $F_{n}$ specifies the alarm frequency at the node $n \in$ NOD. The following additional decision variables are introduced; $y_{a}=1$, if a PSC is settled at the node $a \in$ NOD; or else $0 ; z_{a, e}=1$, if the main road node $e \in E$ is served by the PSC at the node $a \in \mathrm{NOD}$; or else 0 . sall represents the total number of PSCs.

Three types of indices are involved in INO: the node set where the alarms are originated and PSCs are positioned; the set of PSCs; the set of main road nodes. Besides the traveling time matrix among road nodes, for the deployment problem, the alarm frequency of node should be considered. Additionally, the referenced service time promised is introduced as a parameter.

The key of PSC incremental optimization is to determine the quantity of new PSCs and their positions under the criteria of PSC deployment. The variable $y_{a}$ denotes whether a new PSC is settled at the node $a \in N$. Based on the assessment criteria defined above and considering the service time and efficiency, $x_{a, n}$ denotes the assignment of the node $n \in$ NOD to the PSC settled at the node $a \in$ NOD.

According to the assessment criteria for PSCs, six objectives are defined.

(1) The balance of the total working time weighted by the alarm frequency at node is achieved by minimizing the maximal workload of PSC (26).

(2) The weighted response time for node is to be minimized (27).

(3) The total weighted response time is to be minimized (28).

(4) The total number of PSCs is to be minimized (29).

(5) The longest time to blockade the main road nodes is to be minimized (30).

(6) The total time of blockade is to be minimized (31). 
Input $C T_{a, b}$ : the traveling time between two nodes $a \in \mathrm{NOD}$ and $b \in \mathrm{NOD}$;

$C N \subseteq$ NOD: the initial node set anticipating the blockade;

$N$ : The minimal number of nodes anticipating the blockade;

$D I S$ : the minimal length of time between nodes anticipating blockade.

Output $C N \subseteq$ NOD: the node set anticipating the blockade.

Process

Step 1. Calculate the minimal traveling time among the nodes in $C N$ by:

$t=\min _{a, b \in \mathrm{CN}}\left\{C T_{a, b}\right\}$.

Step 2. The termination criterion of the algorithm:

If $(|C N| \leq N) \vee(t \geq D I S)$ Then

\section{Return}

End IF

Step 3. Determine the node to be removed by: $i=\underset{i, a, b \in \mathrm{CN}, i \neq a, i \neq b, a \neq b}{\arg \min }\left\{C T_{i, a}+C T_{i, b}\right\}$.

Step 4. Update the set by: $C N \leftarrow C N \backslash\{i\}$.

Algorithm 1: Node selection for besiege program.

In the following, the constraints of the model INO are formulated ((32)-(48)):

$$
\begin{aligned}
& \min f_{1}=\operatorname{stpma,} \\
& \min f_{2}=n t r m a, \\
& \min f_{3}=n t r a l l \text {, } \\
& \min f_{4}=\text { sall, } \\
& \min f_{5}=\text { etma, } \\
& \min f_{6}=\text { etall, } \\
& n t r_{n}=\sum_{a \in \mathrm{NOD}}\left(x_{a, n} \cdot C T_{a, n}\right), \quad \forall n \in \mathrm{NOD}, \\
& n t r_{n} \leq M T, \quad \forall n \in \mathrm{NOD}, \\
& n t r m a \geq n t r_{n}, \quad \forall n \in \mathrm{NOD}, \\
& \operatorname{stp}_{a}=\sum_{n \in \mathrm{NOD}}\left(x_{a, n} \cdot C T_{a, n} \cdot F_{n}\right), \quad \forall a \in \mathrm{NOD}, \\
& \text { stpma } \geq \operatorname{stp}_{a}, \quad \forall a \in \mathrm{NOD}, \\
& \sum_{a \in \mathrm{NOD}} x_{a, n}=1, \quad \forall n \in \mathrm{NOD}, \\
& x_{a, a}=y_{a}, \quad \forall a \in \mathrm{NOD}, \\
& n \text { trall }=\sum_{n \in \mathrm{NOD}}\left(n t r_{n} \cdot F_{n}\right) \text {, } \\
& y_{a} \cdot M \geq \sum_{n \in \mathrm{NOD}} x_{a, n}, \quad \forall a \in \mathrm{NOD} \\
& y_{s}=1, \quad \forall s \in S \text {, } \\
& \text { sall }=\sum_{a \in \mathrm{NOD}} y_{a} \\
& \sum_{a \in \mathrm{NOD}} z_{a, e}=1, \quad \forall e \in E,
\end{aligned}
$$

$$
\begin{gathered}
\sum_{e \in E} z_{a, e} \leq 1, \quad \forall a \in \mathrm{NOD}, \\
e t_{e}=\sum_{a \in \mathrm{NOD}}\left(z_{a, e} \cdot C T_{a, e}\right), \quad \forall e \in E, \\
\text { etma } \geq e t_{e}, \quad \forall e \in E, \\
\text { etall }=\sum_{e \in E} e t_{e}, \\
y_{a} \geq \sum_{e \in E} z_{a, e}, \quad \forall a \in \mathrm{NOD} .
\end{gathered}
$$

Equations (32)-(39) define the basic constraints about the assignment of the nodes with alarms to the PSCs. Equation (32) defines the response time for each node with alarm. Equation (33) sets the restriction of promised service response time to all nodes, where $M T$ is the length of the promised time. This constraint is hard here, which may make the model infeasible. Therefore, it may be removed in the solving process. The maximal response time for any node is defined by (34) based on (32). Equation (35) computes the workload for each PSC. The maximal workload for all PSCs is defined based on (35). By (36), every node with alarm should be assigned to a PSC. Besides (37), (38) defines the constraint that any PSC at least serves the node where it is settled. In (39), the total weighted response time of all nodes is computed.

The constraints $((40)-(42))$ primarily deal with the restrictions especially for incremental location problem of PSC. In (40), the assignment variable $x$ is employed to denote the location indicator variable $y$, where $M$ is a big number whose minimal value is $|\mathrm{NOD}|$. Because it is the incremental location problem, the node which is already a PSC should be PSC in the new solution (41). The number of PSCs after the incremental optimization is computed by (42).

The constraints in (43)-(48) correlate with blockade. Every main road node should be attached to a PSC to blockade it, as defined in (43), whereas (44) sets the rule that each PSC can only take charge of the blockade of one main road node. The time to blockade any main road node is computed by (45), whereas (46) is then used to define the 


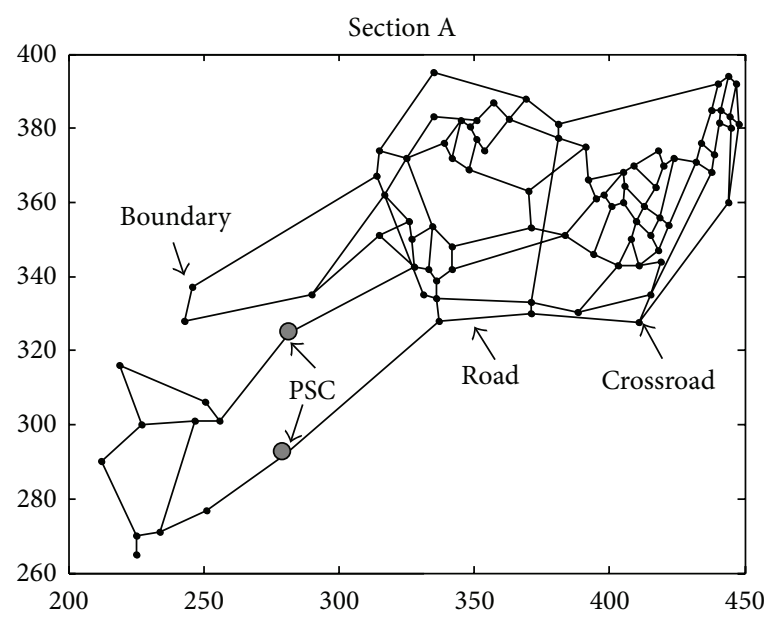

(a) District A

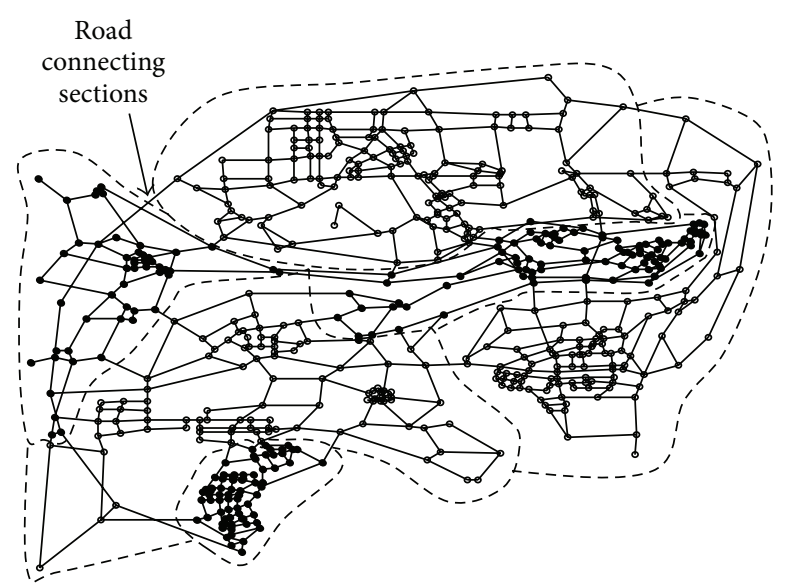

(b) Districts A-F

FIGURE 2: Traffic network and PSC distribution in sample.

maximal time of blockading any node. Then, the total time of blockade is computed by (47). Of course, only the node where a PSC is settled can be sent to blockade a node (48).

3.6. Redeployment Optimization. The main principle of redeployment optimization (REO) is to employ the original set of PSCs as many as possible. In other words, the difference between the original and new set of PSCs should be minimized. Based on INO (Section 3.5), the original set of PSCs is allowed to change so that the constraint in (41) is removed. New PSC can be added, which requires removing the constraint defined by (42). Then, a new objective should be designed to minimize the quantity of PSCs that are settled at new nodes. In other words, the quantity of new PSCs should be minimized:

$$
\min f_{7}=\sum_{a \in \mathrm{NOD} \backslash S} y_{a} .
$$

\section{Case Study}

A network in Figure 2 is used to demonstrate the effects of the above decision models. Because the models built in the study are all ILP or MIP models and the main objective is to build decision models and the general systematic framework for PSC DSS, in the following study, Gurobi 4.6 is employed as a MIP solver to solve the models.

In Figure 2(a), the traffic network of District A with 20 PSCs in a city in China is shown. For the 20 PSCs in District $\mathrm{A}$, there is a coordination problem of how the service areas are probably assigned so that the patrol service can reach the site with contingent event within three minutes (the speed of patrol police car is presumed to be $60 \mathrm{~km} / \mathrm{h}$ ). With the help of the 110 call center and GIS, the position of alarm and the crossroad nearby can be located. The second question is about the emergency blockade to the 13 main crossroads on the boundary of the city by scheduling the 20 PSCs when a contingent event happens. The third question is to determine the quantity and positions of new PSCs to balance the workloads of the current PSCs and shorten the traveling time of patrol services.

Figure 2(b) depicts six sections, A, B, C, D, E, and F. From the view of the assessment and design of all PSCs in the city, the first problem is to assess the present deployment of PSCs. The second problem is what the solution is if it is not rational. The third is the coordination problem among all PSCs in the city. When a serious criminal case occurs at a site in the city, three minutes later the PSCs receive the alarm and the suspect has escaped. To arrest the suspect efficiently, how can the besiege plan be designed and the PSCs be coordinated? This study builds general and extensible models for these problems.

4.1. Service Area Assignment. By GIS, the road network, and PSC deployment data in Figure 2, in the following the above models are demonstrated. In Figure 2(b), there are 582 crossroad nodes, and there are 92 nodes in District A in Figure 2(a). In Table 1, 92 nodes in District A and their frequencies of alarm are listed. The road connection matrix and the distance matrix are ignored in the text. In the city, there are totally 80 PSCs and 20 PSCs in District A. The PSCs and their attached road nodes are listed in Table 2. In Figure 2(b), the quantity of the main nodes on the urban boundary of the city is 17 . When a car goes out of the city, it must pass one of these nodes. In Figure 2(a), 13 nodes are such kind of nodes of District A: 12, 14, 16, 21, 22, 23, 24, 28, 29, 30, 38,48 , and 62 . In addition, there are totally 928 road segments in the city. By these roads, the shortest distance and path between any two nodes can be calculated by Floyd shortest path algorithm. Then, considering the speed of patrol cars, the time matrix can be computed among all nodes. Because the data volume is too larger, the matrix is not included in the paper. The readers who are interested in the dataset can contact the authors to get it.

The SAA (Sections 3.1-3.3) is multiobjective. In the following, the solution of SAA is computed for District A in 
TABLE 1: Positions and alarm frequencies of nodes in District A.

\begin{tabular}{|c|c|c|c|c|c|c|c|c|c|c|c|c|c|c|c|}
\hline Number & $X$ & $Y$ & $F$ & Number & $X$ & $Y$ & $F$ & Number & $X$ & $Y$ & $F$ & Number & $X$ & $Y$ & $F$ \\
\hline 1 & 413 & 359 & 1.7 & 24 & 212 & 290 & 1.1 & 47 & 325 & 372 & 1.6 & 70 & 408 & 350 & 0.9 \\
\hline 2 & 403 & 343 & 2.1 & 25 & 227 & 300 & 1.6 & 48 & 315 & 374 & 1.4 & 71 & 415 & 351 & 1.1 \\
\hline 3 & 383.5 & 351 & 2.2 & 26 & 256 & 301 & 1.2 & 49 & 342 & 372 & 1.2 & 72 & 418 & 347 & 0.8 \\
\hline 4 & 381 & 377.5 & 1.7 & 27 & 250.5 & 306 & 0.8 & 50 & 345 & 382 & 1.1 & 73 & 422 & 354 & 0.9 \\
\hline 5 & 339 & 376 & 2.1 & 28 & 243 & 328 & 1.3 & 51 & 348.5 & 380.5 & 0.8 & 74 & 418.5 & 356 & 1.1 \\
\hline 6 & 335 & 383 & 2.5 & 29 & 246 & 337 & 1.4 & 52 & 351 & 377 & 0.6 & 75 & 405.5 & 364.5 & 0.8 \\
\hline 7 & 317 & 362 & 2.4 & 30 & 314 & 367 & 2.1 & 53 & 348 & 369 & 1.4 & 76 & 405 & 368 & 1.1 \\
\hline 8 & 334.5 & 353.5 & 2.4 & 31 & 315 & 351 & 1.6 & 54 & 370 & 363 & 0.9 & 77 & 409 & 370 & 0.8 \\
\hline 9 & 333 & 342 & 2.1 & 32 & 326 & 355 & 1.5 & 55 & 371 & 353 & 1 & 78 & 417 & 364 & 0.8 \\
\hline 10 & 282 & 325 & 1.6 & 33 & 327 & 350 & 1.4 & 56 & 354 & 374 & 0.5 & 79 & 420 & 370 & 0.8 \\
\hline 11 & 247 & 301 & 2.6 & 34 & 328 & 342.5 & 1.7 & 57 & 363 & 382.5 & 0.8 & 80 & 424 & 372 & 0.8 \\
\hline 12 & 219 & 316 & 2.4 & 35 & 336 & 339 & 1.4 & 58 & 357 & 387 & 1.1 & 81 & 438 & 368 & 1.4 \\
\hline 13 & 225 & 270 & 2.2 & 36 & 336 & 334 & 1.1 & 59 & 351 & 382 & 0.9 & 82 & 438.5 & 373 & 1.1 \\
\hline 14 & 280 & 292 & 2.5 & 37 & 331 & 335 & 0.1 & 60 & 369 & 388 & 0.7 & 83 & 434 & 376 & 0.9 \\
\hline 15 & 290 & 335 & 2.1 & 38 & 371 & 330 & 1.2 & 61 & 335 & 395 & 0.6 & 84 & 438 & 385 & 1 \\
\hline 16 & 337 & 328 & 2.6 & 39 & 371 & 333 & 1.4 & 62 & 381 & 381 & 1.2 & 85 & 440 & 392 & 1.2 \\
\hline 17 & 415 & 335 & 2.5 & 40 & 388.5 & 330.5 & 1.7 & 63 & 391 & 375 & 1.4 & 86 & 447 & 392 & 1.4 \\
\hline 18 & 432 & 371 & 1.9 & 41 & 411 & 327.5 & 1.4 & 64 & 392 & 366 & 0.8 & 87 & 448 & 381 & 1.1 \\
\hline 19 & 418 & 374 & 1.8 & 42 & 419 & 344 & 1.4 & 65 & 395 & 361 & 0.7 & 88 & 444.5 & 383 & 0.9 \\
\hline 20 & 444 & 394 & 1.9 & 43 & 411 & 343 & 1.7 & 66 & 398 & 362 & 0.8 & 89 & 441 & 385 & 1.4 \\
\hline 21 & 251 & 277 & 1.4 & 44 & 394 & 346 & 1.1 & 67 & 401 & 359 & 0.8 & 90 & 440.5 & 381.5 & 0.9 \\
\hline 22 & 234 & 271 & 1.4 & 45 & 342 & 342 & 1.4 & 68 & 405 & 360 & 0.9 & 91 & 445 & 380 & 0.9 \\
\hline 23 & 225 & 265 & 2.4 & 46 & 342 & 348 & 1.2 & 69 & 410 & 355 & 1.1 & 92 & 444 & 360 & 0.8 \\
\hline
\end{tabular}

TABLE 2: Attached nodes of PSCs in District A.

\begin{tabular}{lc}
\hline Number & Node \\
\hline 1 & 1 \\
2 & 2 \\
3 & 3 \\
4 & 4 \\
5 & 5 \\
6 & 6 \\
7 & 7 \\
8 & 8 \\
9 & 9 \\
10 & 10 \\
11 & 11 \\
12 & 12 \\
13 & 13 \\
14 & 14 \\
15 & 15 \\
16 & 16 \\
17 & 17 \\
18 & 18 \\
19 & 19 \\
20 & 20 \\
\hline
\end{tabular}

Figure 2(a). When the objective functions are ignored in the model, it is found that there is no feasible solution due to the constraint defined by (5). The expectation for minimizing the response time can be achieved by minimizing the objectives $f_{1}$ and $f_{2}$. Therefore, in the experiments for SAA, the constraint equation (5) is excluded. The priority order of the three objectives is $f_{2}>f_{3}>f_{1}$, so that goal programming method is employed in the following steps. First, $\min f_{2}$ is taken as the objective to minimize the maximal response time. Then, $n t r m a=8.4$ is returned. A new constraint is added to SAA: $n$ trma $\leq$ 8.4. Second, $\min f_{3}$ is taken as the objective to minimize the total workload. By solving the model, get $n$ trall $=150.7$. The following constraint is also then added to SAA as a new constraint: ntrall $\leq 150.7$. Finally, $\min f_{1}$ is used to balance the workloads. The result is stpma - stpmi $=14.9$.

Therefore, by simple process on the outputs of the solver, the results (Table 3 ) are obtained. The total workload and the served nodes by each PSC are presented (Table 3 ). The rows are sorted by decreasing order of workload, and the workload is normalized after being accumulated (Table 3 ). The results are then incorporated into Figure 3. The former 6 (30\%) PSCs accomplish $50 \%$ of the total workload; the former $10(50 \%)$ PSCs finish about $80 \%$ workload; and the former 14 (70\%) PSCs undertake more than $90 \%$ workload (Figure 3 ). Therefore, the objectives, such as decreasing the alarm response time, enhancing the alarm service quantity, and minimizing the workload, do commonly make the workloads unbalanced between different PSCs. The workloads are mostly served by a few PSCs. Obviously, the quantity of PSCs and their positions impact the service quantity and service efficiency. 
TABLE 3: Total workload and served nodes for every PSC.

\begin{tabular}{lcl}
\hline PSC & $s t p$ & Served nodes by PSC \\
\hline 1 & 9.4 & $1,67,68,69,71,74,75,78$ \\
2 & 13.5 & $2,38,40,43,44,70,72$ \\
3 & 7.7 & $3,54,55,65,66$ \\
4 & 8.6 & $4,57,60,62,63,64$ \\
5 & 6.3 & $5,49,50,52,53$ \\
6 & 11.4 & $6,47,51,56,58,59$ \\
7 & 12.7 & $7,30,31,48,61$ \\
8 & 5.6 & $8,32,33,46$ \\
9 & 4.4 & $9,34,35,36,37$ \\
10 & 0 & 10 \\
11 & 2.8 & $11,26,27$ \\
12 & 3.2 & 12,25 \\
13 & 11.3 & $13,21,22,23,24$ \\
14 & 0 & 14 \\
15 & 14.9 & $15,28,29$ \\
16 & 8.4 & $16,39,45$ \\
17 & 2.8 & $17,41,42$ \\
18 & 10.9 & $18,73,80,81,82,83,84,90$ \\
19 & 3.8 & $19,76,77,79$ \\
20 & 13 & $20,85,86,87,88,89,91,92$ \\
\hline
\end{tabular}

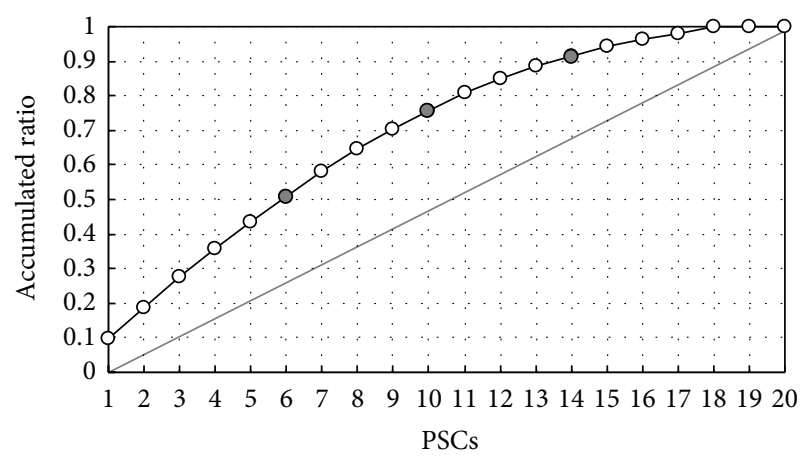

Figure 3: Accumulated workload in decreasing order by PSC.

In Figure 4, according to the results of solving SAA, the ratio of the quantities of nodes and the ratios of the quantities of alarms are analyzed in the cases of different lengths of response time. When the workloads of PSCs are not balanced, the promise of response within three minutes can be ensured for almost 95\% nodes and almost 95\% alarms (Figure 4). Therefore, if the patrol-related resource is enough, at least the present deployment can satisfy the service requirements. However, the unbalanced nature indicates certain waste of patrol-related resources (Figure 4).

4.2. Emergent Blockade. Taking District A in Figure 2(a) as a sample, the emergent blockade solution is studied to schedule 20 PCSs to blockade the 13 main road nodes. According to the two objectives in (13) and (14) and the decision variables and constraints, EMB is a multiobjective MIP model. However, when the resources of PSCs are adequate, the key objective
TABLE 4: Assignment of PSCs to main road nodes.

\begin{tabular}{lccc}
\hline Number & $\begin{array}{c}\text { Main road } \\
\text { node }(e \in E)\end{array}$ & PSC $(s \in S)$ & $\begin{array}{c}\text { Blockade } \\
\text { time }\left(e t_{e}\right)\end{array}$ \\
\hline 1 & 12 & 12 & 0 \\
2 & 14 & 16 & 7 \\
3 & 16 & 9 & 2 \\
4 & 21 & 14 & 4 \\
5 & 22 & 10 & 8 \\
6 & 23 & 13 & 1 \\
7 & 24 & 11 & 4 \\
8 & 28 & 15 & 5 \\
9 & 29 & 7 & 9 \\
10 & 30 & 6 & 4 \\
11 & 38 & 2 & 4 \\
12 & 48 & 5 & 3 \\
13 & 62 & 4 & 1 \\
\hline
\end{tabular}

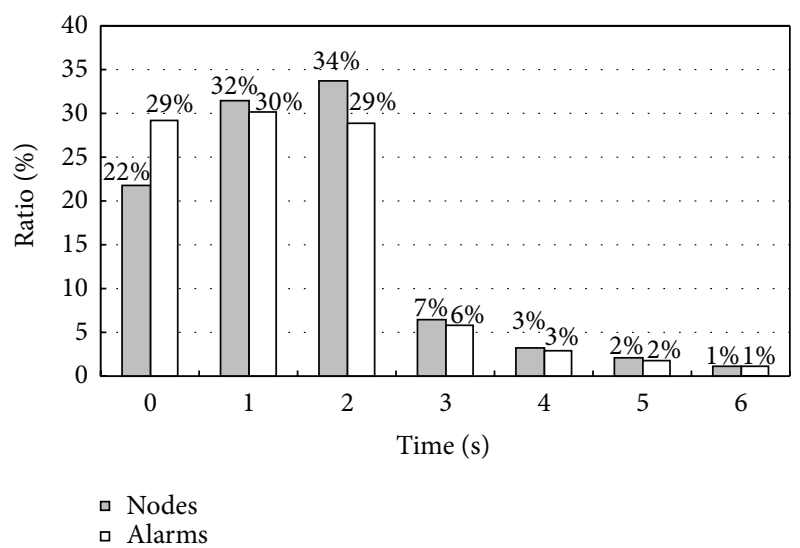

FIGURE 4: Quantity of nodes and alarms for different length of response time.

is to implement a whole blockade for all main road nodes by minimizing $f_{1}$; then the performance of PSC assignment can be further optimized. Therefore, the following steps are conducted to adjust the model and solve it by the dataset of District A (Figure 2(a)). At first, by minimizing the maximal blockade time, $\min f_{1}$, the result etma $=9$ and etall $=76$ is then taken as constraint (50), which is incorporated into the new model. Then, taking $\min f_{2}$ as the objective to minimize the total blockade time, etall $=52$ is returned:

$$
\text { etma } \leq 9 .
$$

The final assignment solution is then shown in Table 4, where the main road nodes, the PSC to blockade this node, and the blockade time are presented.

4.3. Besiege Program. In BEP, $C N$ can be determined by (21), where $C T$ is a parameter. For example, the node set $C N$ 
TABLE 5: Besiege programs.

\begin{tabular}{|c|c|c|c|c|c|c|}
\hline 7 minutes & 8 minutes & 9 minutes & 10 minutes & & 15 minutes & \\
\hline $\begin{array}{l}3 \rightarrow 55 \\
5 \rightarrow 5 \\
6 \rightarrow 6 \\
15 \rightarrow 15 \\
16 \rightarrow 16 \\
171 \rightarrow 232 \\
173 \rightarrow 173\end{array}$ & $\begin{array}{c}1 \rightarrow 3 \\
2 \rightarrow 38 \\
3 \rightarrow 55 \\
4 \rightarrow 54 \\
5 \rightarrow 5 \\
6 \rightarrow 6 \\
10 \rightarrow 10 \\
15 \rightarrow 15 \\
16 \rightarrow 16 \\
171 \rightarrow 231 \\
172 \rightarrow 244 \\
173 \rightarrow 173\end{array}$ & $\begin{array}{c}1 \rightarrow 3 \\
2 \rightarrow 38 \\
3 \rightarrow 55 \\
4 \rightarrow 54 \\
5 \rightarrow 50 \\
6 \rightarrow 6 \\
10 \rightarrow 10 \\
15 \rightarrow 15 \\
16 \rightarrow 560 \\
17 \rightarrow 40 \\
19 \rightarrow 44 \\
168 \rightarrow 60 \\
170 \rightarrow 171 \\
171 \rightarrow 232 \\
172 \rightarrow 231 \\
173 \rightarrow 173\end{array}$ & $\begin{aligned} 1 & \rightarrow 40 \\
2 & \rightarrow 38 \\
3 & \rightarrow 55 \\
4 & \rightarrow 54 \\
5 & \rightarrow 5 \\
6 & \rightarrow 6 \\
10 & \rightarrow 10 \\
15 & \rightarrow 15 \\
16 & \rightarrow 16 \\
17 & \rightarrow 2 \\
18 & \rightarrow 64 \\
19 & \rightarrow 44 \\
168 & \rightarrow 60 \\
170 & \rightarrow 171 \\
171 & \rightarrow 229 \\
172 & \rightarrow 228 \\
173 & \rightarrow 173 \\
174 & \rightarrow 216\end{aligned}$ & $\begin{array}{c}1 \rightarrow 63 \\
2 \rightarrow 1 \\
3 \rightarrow 55 \\
4 \rightarrow 54 \\
5 \rightarrow 5 \\
6 \rightarrow 6 \\
10 \rightarrow 10 \\
11 \rightarrow 26 \\
12 \rightarrow 12 \\
14 \rightarrow 14 \\
15 \rightarrow 15 \\
16 \rightarrow 16 \\
17 \rightarrow 64 \\
18 \rightarrow 62 \\
19 \rightarrow 65 \\
20 \rightarrow 69\end{array}$ & $\begin{array}{c}167 \rightarrow 273 \\
168 \rightarrow 79 \\
169 \rightarrow 240 \\
170 \rightarrow 216 \\
171 \rightarrow 170 \\
172 \rightarrow 219 \\
173 \rightarrow 173 \\
174 \rightarrow 217 \\
175 \rightarrow 190 \\
176 \rightarrow 189 \\
178 \rightarrow 220 \\
179 \rightarrow 222 \\
180 \rightarrow 223 \\
181 \rightarrow 274 \\
182 \rightarrow 214 \\
372 \rightarrow 27\end{array}$ & $\begin{array}{c}373 \rightarrow 21 \\
374 \rightarrow 489 \\
376 \rightarrow 22 \\
383 \rightarrow 11 \\
384 \rightarrow 25 \\
475 \rightarrow 532 \\
476 \rightarrow 557 \\
477 \rightarrow 564 \\
478 \rightarrow 533 \\
480 \rightarrow 551 \\
481 \rightarrow 531 \\
482 \rightarrow 475 \\
484 \rightarrow 529 \\
485 \rightarrow 480\end{array}$ \\
\hline
\end{tabular}

within $C T=10$ minutes' distance from node 32 contains the nodes as shown in the following:

$$
C N=\left\{\begin{array}{c}
2,3,4,5,6,7,8,9,10,15 \\
16,28,30,31,33,34,35,36,37,38 \\
39,40,44,45,46,47,48,49,50,51, \\
52,53,54,55,56,57,58,59,60,61, \\
63,64,65,66,67,171,173,216,228,229,230
\end{array}\right\} .
$$

Setting $T L$ to $7,8,9,10$, and 15 , the besiege programs by BEP can be obtained (Table 5), where " $a \rightarrow b$ " denotes "the node of PSC $\rightarrow$ the blockaded node," which represents the assignment of PSC to the blockaded node.

4.4. Incremental Optimization. The model INO is a multiobjective model with six objectives. This study does not aim at a general algorithm for multiobjective MIP model. In the following, the steps considering the priorities of all objectives are designed to solve the model.

(1) Due to the high cost of building PSC, the quantity of PSCs should be minimized. By taking $\min f_{4}$ as objective, sall $=24$ will be returned, while the alarms from all nodes can be served within 3 minutes. Therefore, a new constraint as (52) is defined for the new model to limit the quantities of PSCs.

(2) Taking $\min f_{5}$ as objective to minimize the longest blockade time for serious contingent accident, etma $=$ 8 is returned. Therefore, a new constraint in (53) is incorporated.

(3) Taking $\min f_{6}$ as objective to minimize the total blockade time, etall $=33$ is returned. Similarly, a new constraint as (54) is obtained.

(4) Taking $\min f_{2}$ as objective to minimize the longest weighted response time, $n t r m a=4.8$ is returned. A new constraint is defined by (55).
(5) Then, taking $\min f_{1}$ as objective to balance the workloads between different PSCs, stpma $=11.3$ is returned and a new constraint is (56).

(6) Finally, taking $\min f_{3}$ as objective to minimize the weighted response time, $n$ trall $=117.1$ is returned, and the final results are shown in Tables 10 and 11.

In Table 6, the PSC, its attached node, its workload, and served nodes are presented. The notation "new" indicates that the PSC is a new one suggested by solving the model INO. On the basis of the original 20 PSCs, four new PSCs are settled at the nodes 29, 38, 48, and 89.

In Table 7, the assigned PSC and the time for blockade are shown when all 13 main road nodes are required to be blockaded.

By removing the constraint defined by (43), INO is then changed to a model that directly locates all PSCs. Moreover, by incorporating the quantity limit of PSCs, the new results (Table 8 ) can be obtained by similar steps proposed above. Besides the solution of 24 PSCs after incremental optimization, two additional solutions with 15 and 16 PSCs are obtained (Table 8). Different quantity of PSCs will produce different values of various criteria (Table 8). However, apparently the solution with more PSCs will show better performances of almost all criteria:

$$
\begin{gathered}
\text { sall } \leq 24, \\
\text { etma } \leq 8, \\
\text { etall } \leq 33, \\
\text { ntrma } \leq 4.8, \\
\text { stpma } \leq 11.3 .
\end{gathered}
$$

4.5. Assessment of the Present PSCs. For the dataset of the entire city with 582 nodes and 80 PSCs, by adjusting the 
TABLE 6: PSC, its workload, and served nodes.

\begin{tabular}{lcl}
\hline PSC & Workload & Nodes \\
\hline 1 & 11 & $1,67,68,69,71,72,74,75,78$ \\
2 & 7.1 & $2,40,43,44,70$ \\
3 & 7.7 & $3,54,55,65,66$ \\
4 & 8.6 & $4,57,60,62,63,64$ \\
5 & 9.4 & $5,49,50,51,52,53,56$ \\
6 & 5.1 & $6,58,59$ \\
7 & 8.3 & $7,30,32,47$ \\
8 & 2.6 & $8,33,46$ \\
9 & 7 & $9,34,35,36,45$ \\
10 & 0 & 10 \\
11 & 2.8 & $11,26,27$ \\
12 & 3.2 & 12,25 \\
13 & 11.3 & $13,21,22,23,24$ \\
14 & 0 & 14 \\
15 & 4.8 & 15,31 \\
16 & 0.2 & 16,37 \\
17 & 2.8 & $17,41,42$ \\
18 & 7.1 & $18,73,80,81,82,83$ \\
19 & 3.8 & $19,76,77,79$ \\
20 & 2.6 & $20,85,86$ \\
\hline 29 (new) & 1.3 & 28,29 \\
38 (new) & 1.4 & 38,39 \\
48 (new) & 1.8 & 48,61 \\
89 (new) & 7.2 & $84,87,88,89,90,91,92$ \\
\hline
\end{tabular}

TABle 7: Main road node, time to blockade it, and the PSC that blockades it.

\begin{tabular}{lcc}
\hline Main road node & Blockade time & PSC \\
\hline 12 & 0 & 12 \\
14 & 7 & 16 \\
16 & 2 & 9 \\
21 & 4 & 14 \\
22 & 8 & 10 \\
23 & 1 & 13 \\
24 & 4 & 11 \\
28 & 5 & 15 \\
29 & 0 & 29 \\
30 & 1 & 7 \\
38 & 0 & 38 \\
48 & 0 & 48 \\
62 & 1 & 4 \\
\hline
\end{tabular}

model in Section 3.4, the assessment is conducted. At first, the original setting of the 80 PSCs is accepted (43). Then, (57) is added to restrict the entrance of new PSCs. Further, the promised response time is adjusted to $M T=30$.

The results (Table 9) present the assessment result of the current PSC configuration and deployment. The values about "blockade time," "response time," and "workload" are a bit bigger than the values in Table 8 . The present solution can be optimized further.
By incremental optimization and direct optimization, new configuration and deployment programs for PSC are studied. The solutions are assessed under the six criteria:

$$
y_{s}=0, \quad \forall s \in \mathrm{NOD} \backslash S .
$$

4.6. Incremental Optimization and Assessment. The first method to improve the PSC system is to add new PSCs. In the following, new PSCs are introduced to satisfy the premised service time. First, (43) is accepted and (57) is removed, so that the model is changed to INO. Second, MT is set to 3 and 5. Then, two solutions are returned with the assessment result (Table 10). When $M T=3,134$ PSCs are required. It costs much because 64 PSCs should be added. However, the assessment result of other criteria all shows that it is really good. When $M T=5$, the quantity of PSCs decreases to 94 and the assessment values are deteriorated rationally.

4.7. Direct Optimization and Assessment. Direct optimization uses a revised version of INO where (43) and (57) are removed. Similarly, according to the steps proposed above, when $M T$ is set to 3, 4, and 5, the assessment result is obtained (Table 11). The solutions cannot be compared directly because multiple criteria are involved. Moreover, the solving strategies also affect the results greatly.

4.8. Redeployment Optimization. When $M T=3$, get sall $=$ 94. Then, by the revised model above, a new set of PSCs can be obtained. By solving the model, $f_{7}=14$ is returned while the original PSCs are all kept in the new solution. When sall = 94 , if it is expected to minimize $n t r a l l$, the result is $f_{7}=60$. Therefore, only 34 original PSCs are kept in the new solution.

\section{Conclusion}

The quantity of patrol service centers and their locations and the scheduling of patrol-related resources are important for urban security and protection. A general framework of PSC DSS and a series of decision models are proposed, which are demonstrated in a city with six districts. From the view of optimizing and scheduling the patrol-related resources, the assessment criteria for PSC deployment are designed, and six models are built. The PSC service area assignment, emergent blockade, and emergent besiege implement the solutions of assignment of PSCs to alarms and dynamic organization of PSCs when contingent events happen. The independent service by a single PSC and the coordinated response by multiple PSCs are both focused on. Based on the incremental optimization model, the general assessment and optimization models are built. The results of the strategies including direct optimization, incremental optimization, and redeployment are analyzed. As an important contribution, the study developed a systematic and holistic integrated solution for the deployment and various important scheduling scenarios of PSCs.

The study involves a wide scope of the models for the operations and management of urban PSCs, whereas only basic deterministic models are examined here. During the 
TABLE 8: Performance comparison of incremental and direct optimization for PSCs.

\begin{tabular}{lcccccc}
\hline Criterion & sall & etma & Etall & ntrma & stpma & ntrall \\
\hline Incremental & 24 & 8 & 33 & 4.8 & 11.3 & 17.1 \\
Direct & 15 & 8 & 45 & 7.5 & 27.6 & 199.3 \\
Direct & 16 & 8 & 24 & 5.2 & 19.1 & 185.1 \\
\hline
\end{tabular}

TABLE 9: Assessment of the PSCs.

\begin{tabular}{lcccccc}
\hline Criterion & sall & etma & etall & ntrma & stpma & ntrall \\
\hline Current situation & 80 & 13 & 93 & 14.4 & 82 & 1367.3 \\
\hline
\end{tabular}

TABLE 10: Incremental optimization for the PSCs.

\begin{tabular}{lccccccc}
\hline Criterion & $M T$ & sall & etma & etall & ntrma & stpma & ntrall \\
\hline Incremental & 3 & 134 & 4 & 8 & 4.8 & 34 & 818.4 \\
Incremental & 5 & 94 & 5 & 29 & 8.5 & 62 & 1168 \\
\hline
\end{tabular}

TABLE 11: Direct optimization of the configuration of PSCs.

\begin{tabular}{lccccccc}
\hline Criterion & $M T$ & sall & etma & etall & ntrma & stpma & ntrall \\
\hline Direct & 3 & 94 & 63 & 550 & 56 & 1316 \\
Direct & 4 & 63 & 50 & 66 & 520 & 11 & 14 \\
Direct & 5 & 45 & 633 & 95 & 170 \\
\hline
\end{tabular}

process of the research, some related subjects are discovered and they warrant further studies. First, in the aspects of determining the quantity and locations of PSCs, the service demands originated by averaged quantities of alarms cannot reflect the uncertainty of the demands. Therefore, the stochastic optimization can be a new direction. Second, the practical PSCs will have different capability and capacity for patrol-related services, which impose additional complexities. Third, the parallel service capability of single PSC is not indicated in the study. In practice, a PSC can deal with many alarms at the same time. During blockade and besiege, a PSC can take charge of multiple nodes. Fourth, the starting position for a service may not be the settled node of the PSC. The patrol policeman can accept demands during patrol processes. Therefore, dynamic routing and real-time scheduling models are topics of practical significance.

\section{Conflict of Interests}

The authors declare that there is no conflict of interests regarding the publication of this paper.

\section{Acknowledgments}

This study is partially supported by the National Nature Science of China (71101088, 71171129, 71390521, and 71471109), the Science Foundation of Ministry of Education of China and Shanghai (20113121120002, 14YZ100, 20123121110004, and 13SG48), and the Science and Technology Commission of Shanghai (14DZ2280200 and 12510501600).

\section{References}

[1] D. Reis, A. Melo, A. L. V. Coelho, and V. Furtado, "Towards optimal police patrol routes with genetic algorithms," in Intelligence and Security Informatics, vol. 3975 of Lecture Notes in Computer Science, pp. 485-491, Springer, Berlin, Germany, 2006.

[2] R. T. Coupe and L. Blake, "The effects of patrol workloads and response strength on arrests at burglary emergencies," Journal of Criminal Justice, vol. 33, no. 3, pp. 239-255, 2005.

[3] S. J. D’Amico, S.-J. Wang, R. Batta, and C. M. Rump, "A simulated annealing approach to police district design," Computers and Operations Research, vol. 29, no. 6, pp. 667-684, 2002.

[4] H. C. W. Lau, G. T. S. Ho, Y. Zhao, and W. T. Hon, "Optimizing patrol force deployment using a genetic algorithm," Expert Systems with Applications, vol. 37, no. 12, pp. 8148-8154, 2010.

[5] Y. Lou, Y. Yin, and S. Lawphongpanich, "Freeway service patrol deployment planning for incident management and congestion mitigation," Transportation Research C: Emerging Technologies, vol. 19, no. 2, pp. 283-295, 2011.

[6] B. B. Keskin, S. R. Li, D. Steil, and S. Spiller, "Analysis of an integrated maximum covering and patrol routing problem," Transportation Research E: Logistics and Transportation Review, vol. 48, no. 1, pp. 215-232, 2012.

[7] Y. Yin, "Optimal fleet allocation of freeway service patrols," Networks and Spatial Economics, vol. 6, no. 3-4, pp. 221-234, 2006.

[8] Y. Yin, "A scenario-based model for fleet allocation of freeway service patrols," Networks and Spatial Economics, vol. 8, no. 4, pp. 407-417, 2008.

[9] R. Pal and K. C. Sinha, "Simulation model for evaluating and improving effectiveness of freeway service patrol programs," Journal of Transportation Engineering, vol. 128, no. 4, pp. 355$365,2002$. 
[10] S. E. Bodily, "Police sector design incorporating preferences of interest groups for equality and efficiency," Management Science, vol. 24, no. 12, pp. 1301-1313, 1978.

[11] J. M. Carroll and P. G. Laurin, "Using simulation to assign police patrol zones," Simulation, vol. 36, no. 1, pp. 1-12, 1981.

[12] G. M. Kern, "Computer simulation model for the study of police patrol deployment," Simulation, vol. 52, no. 6, pp. 226-232, 1989.

[13] K. M. Curtin, K. Hayslett-McCall, and F. Qiu, "Determining optimal police patrol areas with maximal covering and backup covering location models," Networks and Spatial Economics, vol. 10, no. 1, pp. 125-145, 2010.

[14] T. Assunção and V. Furtado, "A heuristic method for balanced graph partitioning: an application for the demarcation of preventive police patrol areas," in Advances in Artificial Intelligence-IBERAMIA 2008, vol. 5290 of Lecture Notes in Computer Science, pp. 62-72, Springer, Berlin, Germany, 2008.

[15] A. Khattak, N. Rouphail, K. Monast, and J. Havel, "Method for priority-ranking and expanding freeway service patrols," Transportation Research Record, vol. 1867, pp. 1-10, 2004.

[16] J. M. Chaiken and P. Dormont, "A patrol car allocation model: background," Management Science, vol. 24, no. 12, pp. 12801290, 1978.

[17] J. M. Chaiken and P. Dormont, "A patrol car allocation model: capabilities and algorithms," Management Science, vol. 24, no. 12, pp. 1291-1300, 1978.

[18] L. C. Green and P. J. Kolesar, "A comparison of the multiple dispatch and $\mathrm{M} / \mathrm{M} / \mathrm{c}$ priority queueing models of police patrol," Management Science, vol. 30, no. 6, pp. 665-670, 1984.

[19] L. V. Green and P. J. Kolesar, "Testing the validity of a queueing model of police patrol," Management Science, vol. 35, no. 2, pp. 127-148, 1989.

[20] S. R. Sacks, "Optimal spatial deployment of police patrol cars," Social Science Computer Review, vol. 18, no. 1, pp. 40-45, 2000. 


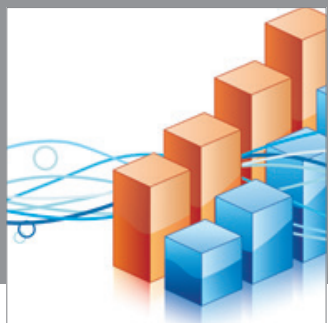

Advances in

Operations Research

mansans

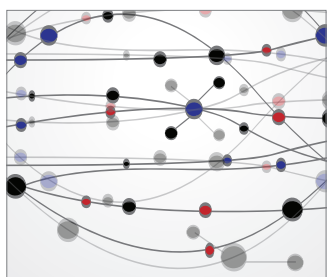

The Scientific World Journal
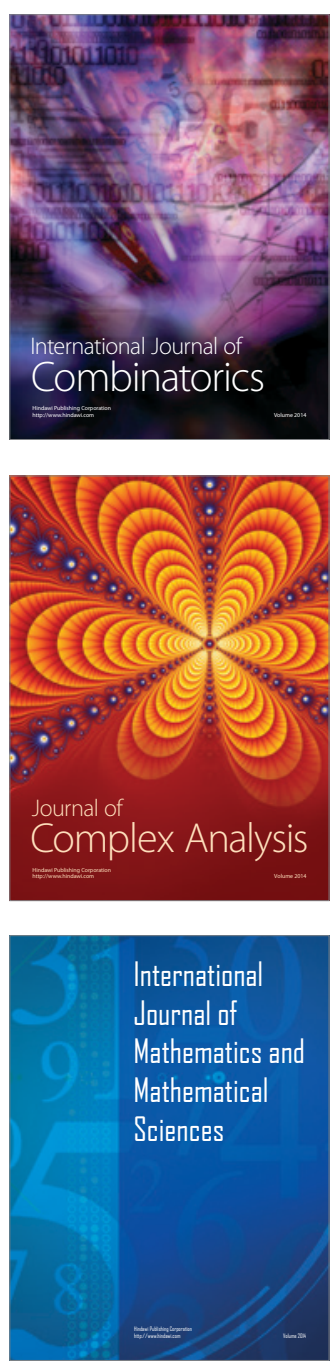
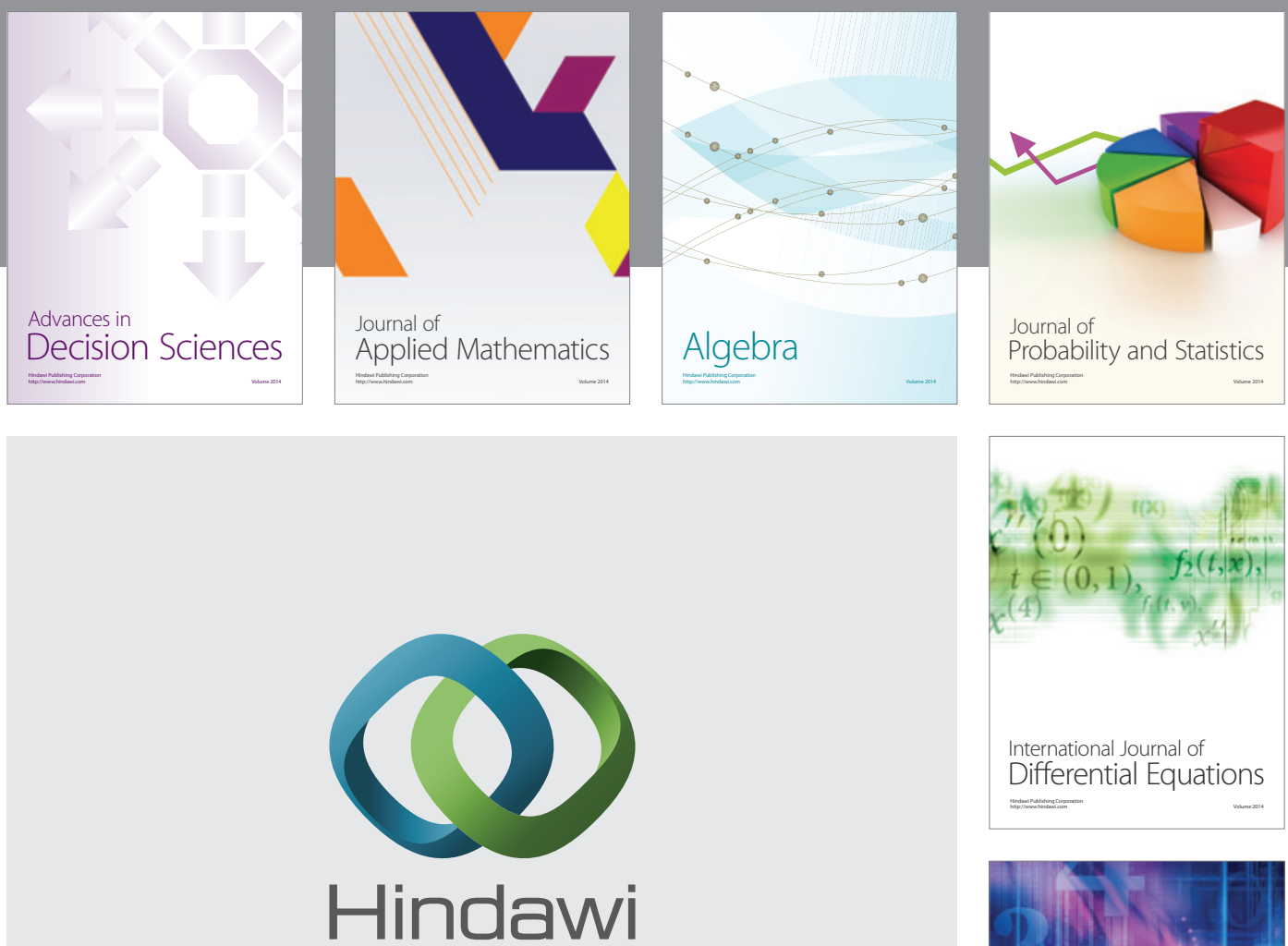

Submit your manuscripts at http://www.hindawi.com
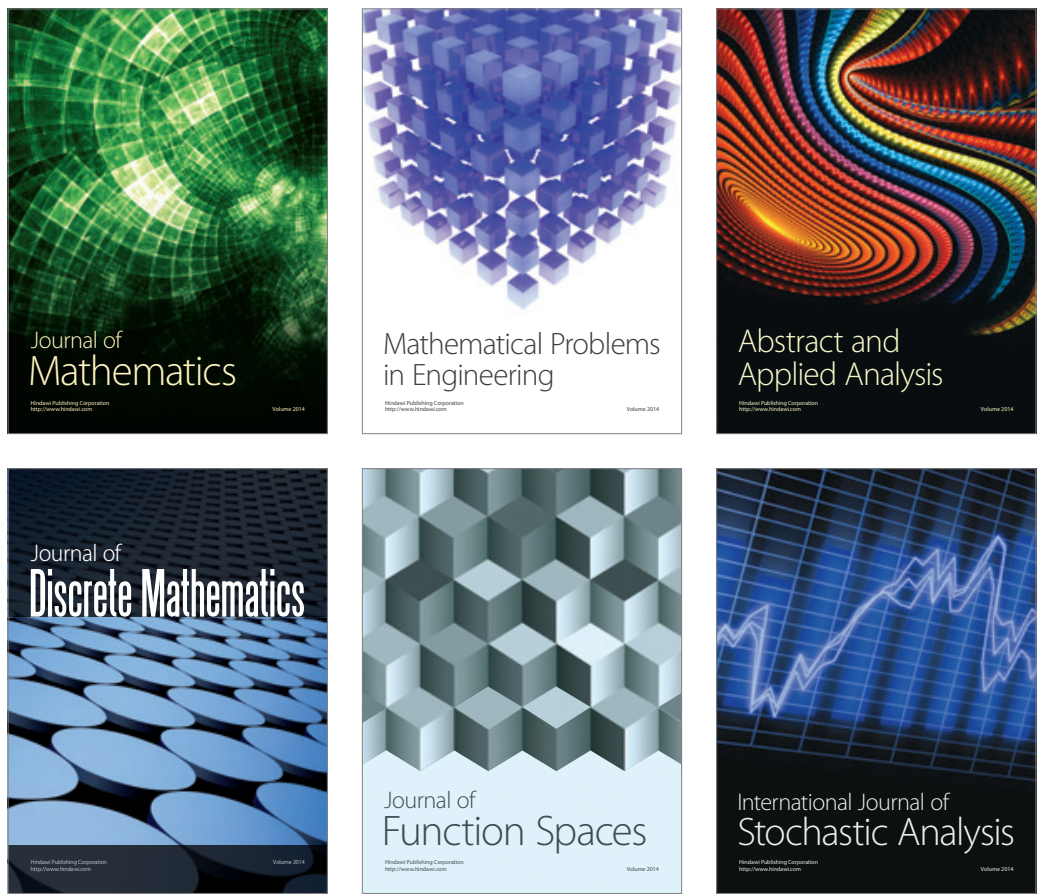

Journal of

Function Spaces

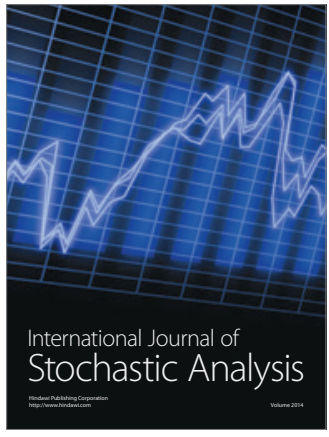

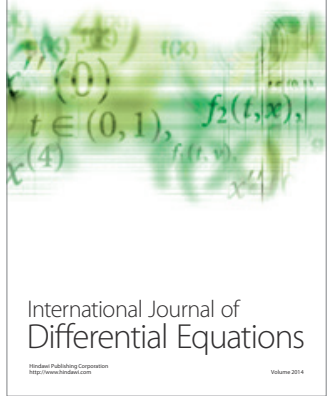
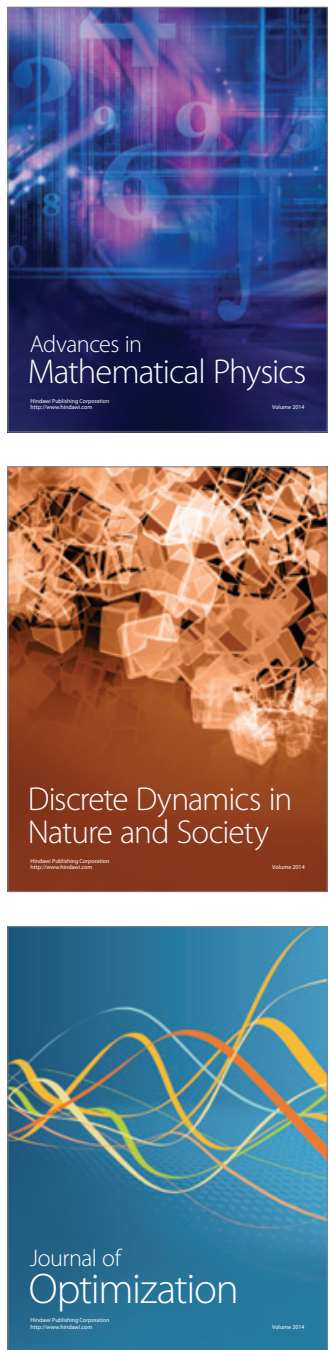